\title{
Study on Synthesis and Properties of Multi-amine Imidazoline as Asphalt Emulsifier
}

\author{
Xiangjun Kong, Chengduo Qian, Yan Lin and Weiyu Fan* \\ State Key Laboratory of Heavy Oil Processing in China University of Petroleum, Qingdao 266580, China \\ Corresponding author
}

\begin{abstract}
Two kinds of multi-amine imidazoline $\mathrm{E}_{1}$ and $\mathrm{E}_{2}$ are synthetized as asphalt emulsifier by the long-chain organic acids and polyethylene polyamine. The purpose of adjusting the mixing time of asphalt emulsion and aggregate can be achieved by controlling the head group number of hydrophilic group. The research results show that the synthetic emulsifier with excellent surface performances; critical micelle concentration $(\mathrm{CMC})$ of $\mathrm{E}_{1}$ and $\mathrm{E}_{2}$ are $0.0728 \mathrm{mmol} . \mathrm{L}^{-1}$ and $0.1508 \mathrm{mmol} . \mathrm{L}^{-1}$ under $25{ }^{\circ} \mathrm{C}$, respectively. The surface tension $\sigma_{\mathrm{cmc}}$ are $23.47 \mathrm{mN} . \mathrm{m}^{-1}$ and $28.50 \mathrm{mN} \cdot \mathrm{m}^{-1}$ under the CMC. The addition of the head group of hydrophilic group makes the CMC and $\sigma_{\mathrm{cmc}}$ increase. The particles of asphalt emulsions prepared by two kinds of emulsifiers are uniform. The volume average particle size are respectively $2.56 \mu \mathrm{m}$ and $2.33 \mu \mathrm{m}$. The increase of amidogen head group number of hydrophilic group is conducive to extend mixing time. the mixing time with the aggregate of the asphalt emulsion prepared by E1 and E2 are respectively $65 \mathrm{~s}$ and $146 \mathrm{~s}$.
\end{abstract}

KEYWORD: imidazoline, emulsifier, mixing time, surface activity.

\section{INTRODUCTION}

The emulsified asphalt technology is one of the important means of road construction and maintenance. Compared with the hot asphalt technology, the emulsified asphalt technology are utilized to pave the road, which saves energy and resources, extends the construction season, and decreases the environmental pollution, etc., therefore, the development of emulsified asphalt has good economic, social and environmental benefits, with great market value(European Commission, 2011). The emulsified asphalt has many application modes in the field of road construction, and different construction modes put forward different requirements for construction and pavement performances of emulsified asphalt(J. Kennedy, 1997, Ashrawi S S1994). For example, rapid cracking asphalt emulsion is utilized in chip seal and grouted macadam, while tack coat is convenient to use rapid or medium setting asphalt emulsion, and slow setting asphalt emulsion are adopted in slurry seal and microsurfacing. The mixing time of asphalt emulsion and aggregate directly influences the construction performance of asphalt emulsion, which concerns construction quality and advantages and disadvantages of subsequent pavement performance, and mixing time is closely related to the structure of asphalt emulsifier(M. Miljković, 2014). The uncontrollable mixing time, poor adaptability of different asphalt and aggre- gate and other problems universally existed in currently applied asphalt emulsion, which seriously influence the application and construction quality of asphalt emulsion. This paper based on the structure design, two kinds of asphalt emulsifier whose hydrophilic group are multi-amine group are synthesized, and the mixing time of asphalt emulsion and aggregate are adjusted by controlling the number of head group in hydrophilic group, thus the requirements of different construction technologies can be met.

\section{EXPERIMENT}

\subsection{The experimental reagent and instrument}

Reagent: diethylenetriamine, tetraethylenepentamine, long chain organic acid, hydrochloride, xylene, anhydrous ethanol, all are analytical pure, production of Sinopharm Chemical Reagent Co., Ltd; heavy traffic AH-70 asphalt.

Instruments: PHS-3C pH meter; HerberPink colloid mill; TX-500C interface tensiometer; LS-POP laser particle size analyzer; YS-2 fluorescent microscope. 


\subsection{The synthesis and surface activity determination of multi-amine imidazoline}

The synthetic method was supported by the available literatures (Fei F L, 2014). Organic acid and amine in a molar ratio of 1:1.05 were added to a reactor, and then a certain amount of water-carrying agent, the dimethylbenzene, was added and mixed for heating and backflow. The amidation reaction was occurred under reflux temperature for a certain period of time; subsequently, the dimethylbenzene was distilled off and evacuated to the vacuum pressure of $0.096 \mathrm{MPa}$, cyclization reaction was then conducted when the increase of temperature to $240{ }^{\circ} \mathrm{C}$. Crude product of imidazoline was obtained. Rotary evaporation was performed under $60^{\circ} \mathrm{C}$ for $1 \mathrm{~h}$ for the removal of water and solvent, and the final product was acquired. Asphalt emulsifiers made from the synthesis of long chain organic acids with diethylenetriamine and tetraethylenepentamine were labeled as $\mathrm{E}_{1}$ and $\mathrm{E}_{2}$, respectively. The reaction equation was shown in Figure 1, in which the $\mathrm{m}$ of emulsifier $\mathrm{E}_{1}$ and emulsifier $\mathrm{E}_{2}$ was 1 and 3, respectively.

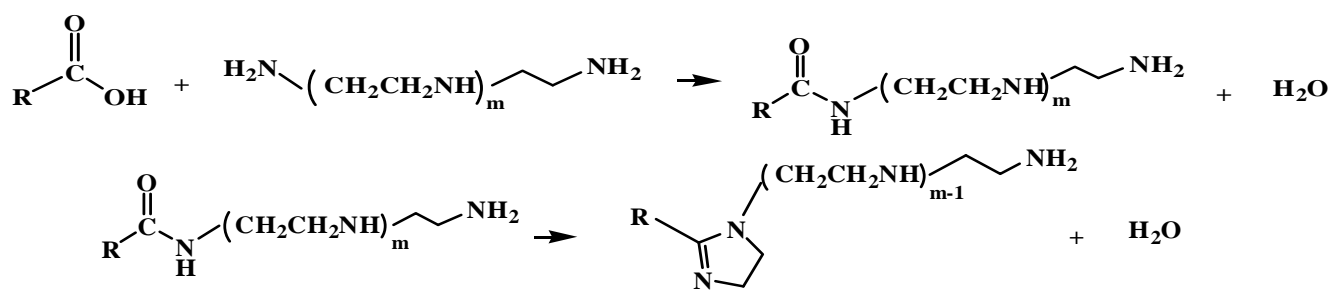

Figure 1 Schematic of synthetic steps (a: acylation, b: cyclization)

The imidazoline surfactant aqueous solutions with different concentration are prepared. TX-500C interface tensiometer are used to determine surface tension under $25^{\circ} \mathrm{C}$. Surface tension as a function of common logarithm of concentration $\operatorname{lgC}$ are drawn, and CMC and $\sigma_{\mathrm{cmc}}$ are determined by inflection point of the curve.

\subsection{The preparation and performance evaluation of asphalt emulsion}

The emulsifier aqueous solution with a certain concentration is prepared and heated to $40 \sim 60^{\circ} \mathrm{C}$, then stirred to dissolve it as the $\mathrm{pH}$ value is adjusted to 2 , and the SBR latex are added. A certain asphalts are weighed (account for the $60 \mathrm{wt} \%$ quality of asphalt emulsion), heated to $135{ }^{\circ} \mathrm{C}$. The colloid mill is launched and the emulsifier aqueous solution are injected into the colloid mill. Then slowly pour the asphalt into the colloid mill to shear. The asphalt emulsion are obtained after emulsifying one minute.

The LS-POP type laser particle size analyzer is used to determine particle size distribution of asphalt emulsion. The YS-2 type fluorescent microscope is used to amplify a certain times of the image for emulsion particles and fluorescent microscopic can be analyzed.

The prepared asphalt emulsion are made storage stability test by reference to The Ministry of Communications JTJ052-2000 "Testing Regulations of Highway Engineering Asphalt and Asphalt Mixture"; the demulsification rate of asphalt emulsion and mixing time of mineral aggregate are determined.

\section{RESULTS AND DISCUSSION}

\subsection{The surface activity of multi-amine imidazoline}

The surface tension of $E_{1}$ and $E_{2}$ change along with the concentration under $25{ }^{\circ} \mathrm{C}$, whose changing curve is shown in Fig.2. The critical micelle concentration (CMC) and $\sigma_{\mathrm{cmc}}$ determined by curve inflection point is shown in Table 1. It can be seen from the Fig.2, along with the increase of concentration, the surface tension of the solution is sharply reduced. When the concentration continues to increase after a certain value, the surface tension will no longer change or change very small. At this time, the surfactant begin to form micelles in solution, and the concentration is CMC. It can be seen from Table 1 that the two kinds of synthesized asphalt emulsifier $\mathrm{E}_{1}, \mathrm{E}_{2}$ can reduce surface tension to $30 \mathrm{mN} \cdot \mathrm{m}^{-1}$, whose CMC are both lesser than $0.16 \mathrm{mmol} . \mathrm{L}^{-1}$, indicating that these two kinds of asphalt emulsifiers both have good surface activity. Compared with $\mathrm{E}_{1}$, as a result of increase of head group number of the hydrophilic group, which cause enhanced hydrophilicity of surfactant monomer structure, solubility of $\mathrm{E}_{2}$ are increased in water, then the driving force of micelle are reduced, therefore, $\mathrm{CMC}$ rise. The increase of the hydrophilic group, volume and area, leading to the lower of the adsorption quantity at the interface of water and air, the surface tension becomes higher.

\subsection{The properties of asphalt emulsion}

The storage stability and microstructure of asphalt emulsion

The synthesized emulsifier $\mathrm{E}_{1}$ and $\mathrm{E}_{2}$ are used to prepare asphalt emulsion, respectively, under the 
conditions: asphalt is AH-70, emulsifier usage $1.5 \mathrm{wt} \%$, asphalt content $60.0 \mathrm{wt} \%$, asphalt temperature $135.0^{\circ} \mathrm{C}$, emulsifier aqueous solution temperature $55.0^{\circ} \mathrm{C}, \mathrm{pH} 2.0$, the properties of the prepared emulsions are shown in Table 2, and fluorescent microscope photos are shown in Fig.3.

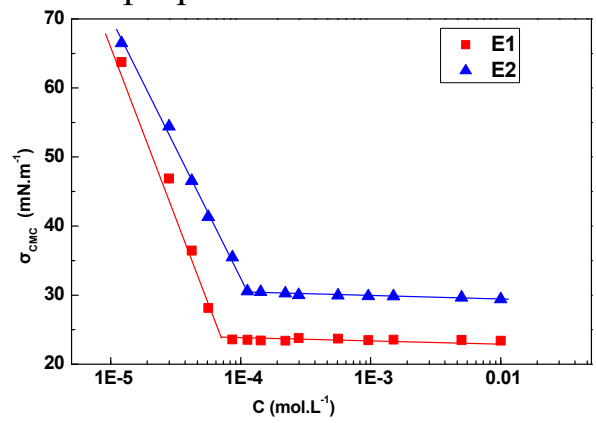

Figure 2 The $\sigma-\operatorname{lgC}$ curve of multi-amine imidazoline

Table 1 The surface activity of multi-amine imidazoline asphalt emulsifier

\begin{tabular}{ccc}
\hline Emusifier & $\mathrm{CMC} /\left(\mathrm{mmol} \cdot \mathrm{L}^{-1}\right)$ & $\sigma \mathrm{CMC} /\left(\mathrm{mN} \cdot \mathrm{m}^{-1}\right)$ \\
\hline $\mathrm{E}_{1}$ & 0.0728 & 23.47 \\
$\mathrm{E}_{2}$ & 0.1508 & 28.50 \\
\hline
\end{tabular}

Table 2 The performance of asphalt emulsions

\begin{tabular}{ccccccc}
\hline \multirow{2}{*}{ Emulsifier } & \multirow{2}{*}{ Engler viscosity(E25) } & \multicolumn{3}{c}{ Storage stability } & \multicolumn{3}{c}{ Particle diameter/ $\mu \mathrm{m}$} \\
\cline { 3 - 7 } & & $1 \mathrm{~d} / \%$ & $5 \mathrm{~d} / \%$ & $\mathrm{D}(4,3)$ & $\mathrm{D} 50$ & $\mathrm{D} 90$ \\
\hline E1 & 11.57 & 0.58 & 2.94 & 2.56 & 2.67 & 3.81 \\
E2 & 10.83 & 0.18 & 0.84 & 2.33 & 2.16 & 4.03 \\
\hline
\end{tabular}

It can be seen from Table 2 that asphalt emulsions prepared by two kinds of emulsifier $\mathrm{E}_{1}$ and $\mathrm{E}_{2}$, with moderate Engler viscosity and good storage stability, can meet the requirements of technical indicators. From the point of particle size distribution, volume average particle size $\mathrm{D}(4,3)$ are both less than $3 \mu \mathrm{m}$.
It can be seen from the fluorescent microscope photos in Fig. 3 that particle size of asphalt emulsion is small and its distribution is uniform, without gathering of large particles, so the stability of the asphalt emulsion is better.
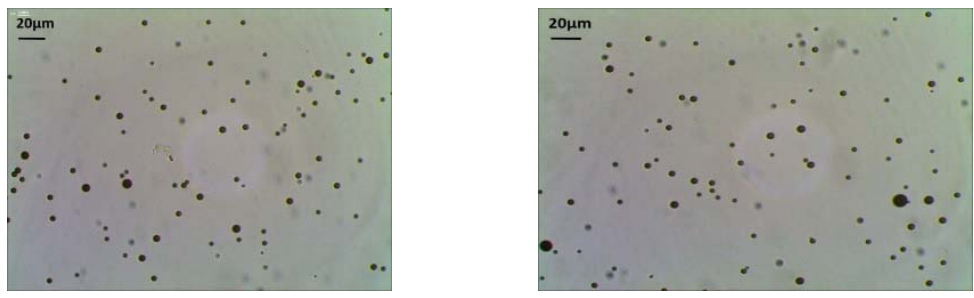

Figure 3 The fluorescent microscopic photos of asphalt emulsions prepared by E1 (a) and E2 (b)

oil vs aggregated rock (asphalt accounted for the pro-

Demulsification rate and mixing time of asphalt emulsion portion of mineral aggregate) $7.2 \mathrm{wt} \%$, fillers (including mineral aggregate) $2.0 \mathrm{wt} \%$, additional water amount (mass percent accounted for mineral aggregate) $6.0 \mathrm{wt} \%$, mixing experimental temperature $25.0^{\circ} \mathrm{C}$. Demulsification rate and mixing time of the two asphalt emulsion are shown in Table 3 adopted to perform the mixing experiment with the prepared asphalt emulsion, according to specification of JTGF40-2004. The test condition are: the ratio of

Table 3 The demulsification classification and mixing time of asphalt emulsions

\begin{tabular}{ccc}
\hline Emulsifier & Demulsification classification & Mixing time/s \\
\hline $\mathrm{E}_{1}$ & Medium-Setting & 65 \\
$\mathrm{E}_{2}$ & Slow-Setting & 146 \\
\hline
\end{tabular}


The mixing time of the asphalt emulsion and aggregate prepared by $E_{2}$ is greater than $E_{1}$ in the same mixing experiment condition, which can be seen from Table 3, namely, the number of head group in hydrophilic group increase, which is beneficial to extend the mixing time. This is because when the hydrophilic group are multi-amine group, head group of hydrophilic group are increased and the formed hydration layer is more solid. When asphalt emulsion and stone mixing, the interfacial film is not easy to burst. Second, when the asphalt emulsion contacts the stone, cationic charge in part of amidogen are neutralized, and the rest head group still preserve cationic. At this time, due to repulsive force of coulomb static electricity, preferentially adsorbed emulsifier molecule in the stone surface and emulsifier molecule in particle interfacial film of asphalt emulsion produce repulsion, thus the contact process is effectively delayed between emulsifier polar groups and stone, so the number of hydrophilic group amidogen is increased, which is good for extend mixing time.

\section{CONCLUSION}

The asphalt emulsifier $E_{1}$ and $E_{2}$ are synthesized by long chain organic acids and polyethylene polyamine, whose hydrophilic group are multi-amine group, with excellent surface performances. The CMC of $\mathrm{E}_{1}$ and $\mathrm{E}_{2}$ are $0.0728 \mathrm{mmol} . \mathrm{L}^{-1}$ and $0.1508 \mathrm{mmol}^{-\mathrm{L}^{-1}}$ under $25{ }^{\circ} \mathrm{C}$, respectively. $\sigma_{\mathrm{cmc}}$ are $23.47 \mathrm{mN} . \mathrm{m}^{-1}$ and 28.50 $\mathrm{mN} . \mathrm{m}^{-1}$. The addition of the head group of hydrophilic group makes CMC increased, but the $\sigma_{c m c}$ lower. The particles of asphalt emulsion prepared by $E_{1}$ and $E_{2}$ are uniform and exquisite, with moderate Engler viscosity and good storage stability. The number of amidogen head group in hydrophilic group increases, which delays contact between asphalt emulsion and aggregates. The mixing time of asphalt emulsion prepared by $\mathrm{E}_{2}$ is obviously longer than asphalt emulsion prepared by $E_{1}$. The purpose of adjusting mixing time of asphalt emulsion and aggregate can be achieved by controlling the number of head group of hydrophilic group.

\section{REFERENCES}

Ashrawi S S. Generating bitumen-in-water dispersions and emulsions: US, US5282984(P). 1994.

European Commission, White Paper on transport, Publications Office of the European Union, Luxembourg, 2011.

Fei F L, Hu J, Wei J X, et al. Corrosion performance of steel reinforcement in simulated concrete pore solutions in the presence of imidazoline quaternary ammonium salt corrosion inhibitor(J). Construction and Building Materials, 2014, $70: 43-53$

J. Kennedy, Alternative Materials and Techniques for Road Pavement Construction, DOE Energy Efficiency Office, London, 1997.
M. Miljković, M. Radenberg, Characterising the influence of bitumen emulsion on asphalt mixture performance, Mater. Struct. 2014, (48): 2195-2210 